\title{
Secretion of the chlamydial virulence factor CPAF requires the Sec-dependent pathway
}

\author{
Ding Chen, ${ }^{1}$ Lei Lei, ${ }^{1}$ Chunxue Lu, ${ }^{1}$ Rhonda Flores, ${ }^{1}$ Matthew P. DeLisa, ${ }^{2}$ \\ Tucker C. Roberts, ${ }^{3}$ Floyd E. Romesberg ${ }^{3}$ and Guangming Zhong ${ }^{1}$
}

\begin{abstract}
Correspondence
Guangming Zhong

Zhongg@uthscsa.edu
\end{abstract}

Received 13 April 2010

Revised 25 May 2010

Accepted 1 June 2010

\author{
${ }^{1}$ Department of Microbiology and Immunology, University of Texas Health Science Center at San \\ Antonio, 7703 Floyd Curl Drive, San Antonio, TX 78229, USA \\ ${ }^{2}$ Department of Biomedical Engineering, Cornell University, Ithaca, NY 14853, USA \\ ${ }^{3}$ Department of Chemistry, The Scripps Research Institute, 10550 N. Torrey Pines Rd, La Jolla, \\ CA 92037, USA
}

The chlamydial protease/proteasome-like activity factor (CPAF) is secreted into the host cytosol to degrade various host factors that benefit chlamydial intracellular survival. Although the fulllength CPAF is predicted to contain a putative signal peptide at its $\mathrm{N}$ terminus, the secretion pathway of CPAF is still unknown. Here, we have provided experimental evidence that the $\mathrm{N}$ terminal sequence covering the M1-G31 region was cleaved from CPAF during chlamydial infection. The CPAF N-terminal sequence, when expressed in a phoA gene fusion construct, was able to direct the export of the mature PhoA protein across the inner membrane of wild-type Escherichia coli. However, E. coli mutants deficient in SecB failed to support the CPAF signalpeptide-directed secretion of PhoA. Since native PhoA secretion was known to be independent of SecB, this SecB dependence must be rendered by the CPAF leader peptide. Furthermore, lack of SecY function also blocked the CPAF signal-peptide-directed secretion of PhoA. Most importantly, CPAF secretion into the host cell cytosol during chlamydial infection was selectively inhibited by an inhibitor specifically targeting type I signal peptidase but not by a type III secretionsystem-specific inhibitor. Together, these observations have demonstrated that the chlamydial virulence factor CPAF relies on Sec-dependent transport for crossing the chlamydial inner membrane, which has provided essential information for further delineating the pathways of CPAF action and understanding chlamydial pathogenic mechanisms.

\section{INTRODUCTION}

Members of the genus Chlamydia share an obligate intracellular life cycle with a distinct biphasic stage (Hackstadt et al., 1997). Host inflammatory responses triggered by chlamydial intracellular survival and replication are thought to contribute to chlamydia-induced pathologies (Stephens, 2003). For example, invasion of and replication in ocular or urogenital epithelial tissues by Chlamydia trachomatis can trigger inflammatory responses that may lead, respectively, to either trachoma in the eye (Mabey, 2008) or urogenital tract complications such as pelvic inflammatory diseases, ectopic pregnancy and infertility (Mårdh, 2004). Infection of respiratory epithelial

Abbreviations: CPAF, chlamydial protease/proteasome-like activity factor; CPAFc, CPAF C terminus; CPAFn, CPAF $N$ terminus; DMEM, Dulbecco's modified Eagle medium; EB, elementary body; FBS, fetal bovine serum; HMM, hidden Markov model; HRP, horseradish peroxidase; $\mathrm{mAb}$, monoclonal antibody; MBP, maltose binding protein; NN, neural networks; OMV, outer membrane vesicle; $\mathrm{RB}$, reticulate body; SRP, signal recognition particle. cells with Chlamydia pneumoniae not only leads to various airway inflammatory pathologies but may also exacerbate pathologies elsewhere, including the vessel wall tissues (Campbell \& Kuo, 2004). Thus, understanding how chlamydial organisms maintain their intracellular survival and replication should advance our knowledge of chlamydial pathogenic mechanisms.

Chlamydia has to overcome many obstacles to establish a successful intracellular infection. First, the infectious elementary body (EB) has to enter a non-phagocytic epithelial cell, which is probably achieved by chlamydial injection of a protein into the host cell to induce phagocytosis (Clifton et al., 2004). Second, once inside a host cell, the chlamydia-laden vacuole (also called inclusion) has to prevent fusion with host cell lysosomes (Eissenberg et al., 1983) when the intravacuolar EB differentiates into a reticulate body (RB) for biosynthesis and replication. Third, to grow inside the inclusion, chlamydial organisms have to import nutrients and possibly energy from the host cell ( $\mathrm{Su}$ et al., 2004). 
Fourth, chlamydial organisms must maintain the integrity of the infected cell while RBs are still undergoing replication (Fan et al., 1998; Greene et al., 2004; Xiao et al., 2005). Finally, once the progeny RBs differentiate back to the infectious EBs, how to safely exit the infected cell to infect neighbouring cells is another challenge facing the chlamydial organisms (Hybiske \& Stephens, 2007). One of the chlamydial strategies for dealing with these growth obstacles is to secrete various proteins into the inclusion membrane (Li et al., 2008a; Rockey et al., 1995; Rzomp et al., 2006) and into the host cell cytosol (Zhong et al., 2001).

Among the chlamydial secreted proteins that can be visualized in the cytoplasm of the infected cells are chlamydial protease/proteasome-like activity factor (CPAF) (Fan et al., 2002; Zhong et al., 2001; Zhong, 2009), pgp3 (Li et al., 2008b), CT621 (Hobolt-Pedersen et al., 2009), Cpn0796 (Vandahl et al., 2005) and Cpn0797 (Dong et al., 2006). The function of CPAF has been extensively studied (Huang et al., 2008; Zhong et al., 2001). CPAF is a powerful serine protease with a water-mediated catalytic triad consisting of H105, S499 and E558 (Huang et al., 2008), which is capable of degrading a wide spectrum of host proteins. CPAF degrades transcription factors such as RFX5 (Zhong et al., 2000) and USF1 (Zhong et al., 1999), which are required for activation of antigen presentation genes that may aid in chlamydial evasion of host immune recognition. CPAF also digests $\mathrm{BH} 3$-only proteins that are responsible for detecting intracellular stress signals, to potentially help chlamydial evasion of host cell apoptosis triggered by chlamydial intracellular growth (Pirbhai et al., 2006). Finally, CPAF can cleave cytoskeleton proteins to facilitate/promote chlamydial inclusion expansion and stability (Dong et al., 2004c; Kumar \& Valdivia, 2008). CPAF is synthesized as an inactive proform and processed into two mature fragments [designated CPAF $\mathrm{N}$ terminus (CPAFn) and CPAF C terminus (CPAFc)] (Dong et al., 2004a). CPAFn and CPAFc form an intramolecular heterodimer. Both cleavage and intramolecular dimerization are required for CPAF proteolytic activity (Dong et al., 2004b). Recent crystal structural data has revealed that a fully active CPAF is a homodimer of two CPAFn/c heterodimers (CPAFn/ c:CPAFn/c; Huang et al., 2008). Despite the extensive knowledge about CPAF function and biochemical properties, it is still unclear how CPAF is secreted from chlamydial organisms into the host cell cytosol.

The chlamydial genome encodes many of the key components required for both type II and type III secretion systems (http://stdgen.northwestern.edu/). There are at least ten operons coding for chlamydial type III secretion system-related molecules (Hefty \& Stephens, 2007). A functional chlamydial type III secretion system has been identified (Fields \& Hackstadt, 2000; Fields et al., 2003; Spaeth et al., 2009) and many effector molecules have been determined (Betts et al., 2009; Spaeth et al., 2009). However, increasing studies have shown that the Secdependent pathway may also be required for secretion of certain chlamydial effectors and that this may be essential in bacterial pathogenesis (Lee \& Schneewind, 2001; Pugsley et al., 1990, 2004). Generally, proteins with N-terminal signal peptides are substrates secreted by the Sec-dependent pathway (Stathopoulos et al., 2000). The cytosolic precursor proteins are recognized by either the secretiondedicated chaperone SecB (Francetic \& Kumamoto, 1996; Zhou \& Xu, 2005) or the signal recognition particle (SRP) that can act as a GTP-dependent chaperone and target the substrate to a SecYEG translocase complex in the cytoplasmic or inner membrane (Baba et al., 1990; Collinson, 2005) for access to the periplasmic space (Collinson, 2005; Driessen \& Nouwen, 2008; Economou, 1999). SecY is absolutely required for the translocase activity while SecE and SecG are auxiliary molecules (Saier, 2006). An ATPdependent motor protein SecA is associated with the SecYEG complex on the cytoplasmic side to promote the access of the chaperone-assisted substrate proteins to the inner membrane SecYEG complex. After crossing the inner membrane, the N-terminal signal peptides are cleaved by signal peptidases LepB or LspA so that the substrate protein can be fully released into the periplasm (Dalbey, 1991; Paetzel et al., 2002; Tuteja, 2005). The periplasmlocalized substrate protein can further exit the bacterial organisms by passing through the outer membrane via the type II machinery formed in part by the general secretion protein D (GspD). Other Gsps such as GspE and GspF may help the periplasmic substrate protein engage the outer membrane GspD pore (Lee \& Schneewind, 2001). The chlamydial genome encodes many homologues of the key components required for a functional type II secretion pathway (http://stdgen.northwestern.edu/). The chlamydial homologue of the essential translocase subunit SecY is encoded by the $C$. trachomatis ORF CT510; the homologue of SecF is CT448. Although there is no SecB homologue, chlamydia does encode many general chaperones including three GroELs (CT110, CT604 and CT755; Karunakaran et al., 2003). Some of these chaperones may help secretion by interacting with two chlamydial homologues of SecA (CT141 and CT701) to bring the substrate protein to the chlamydial SecYEG translocon. In addition, the chlamydial FtsY (an SRP receptor-like protein) may act as a GTPdependent chaperone that promotes interaction between the substrate protein and the SecYEG translocon. The chlamydial homologues of signal peptidases LepB and LspA are CT020 and CT408, respectively. Finally, the chlamydial homologue of GspD (CT572) is predicted to form an outer membrane pore that facilitates secretion of periplasmic substrate proteins out of the chlamydial organisms. The chlamydial homologues of GspE and GspF (CT571 and CT570) may further facilitate the extracellular localization. Thus, chlamydia possesses homologues of all the key components of the Sec-dependent pathway. However, there has been no direct experimental evidence to demonstrate the functionality, probably due to a lack of genetic tools for manipulating the chlamydial genome or reagents required for blocking Sec-dependent protein export. 
Although CPAF is also predicted to carry an N-terminal signal peptide, there has been no experimental data to demonstrate the secretion pathway of CPAF. In the current study, we have provided direct experimental evidence to identify the CPAF secretion pathway. We have found that the N-terminal sequence covering the methionine in position 1 (M1) to glycine in position 31 (G31) is cleaved from CPAF during chlamydial infection, and the putative CPAF signal peptide was able to direct the export of a heterologous substrate protein into the bacterial periplasmic space. More importantly, the functionality of the CPAF N-terminal signal peptide is dependent on both SecB and $\mathrm{SecY}$ as assayed in Escherichia coli. Finally, we found that CPAF secretion into the host cell cytosol during chlamydial infection can be blocked by an inhibitor specifically targeting signal peptidase but not by a type III secretion system-specific inhibitor. Together, these observations demonstrate for the first time that the chlamydial Sec-dependent pathway is functional and that CPAF secretion requires the Sec-dependent pathway.

\section{METHODS}

Cell culture and chlamydial infection. HeLa229 cells (human cervical epithelial cell line, ATCC) were grown in Dulbecco's modified Eagle medium (DMEM; Invitrogen) supplemented with $10 \%$ fetal bovine serum (FBS; Atlanta Biologicals) in a humidified incubator in the presence of $5 \% \mathrm{CO}_{2}$. C. trachomatis serovar LGV2 $(\mathrm{L} 2 / 434 / \mathrm{Bu})$ or $\mathrm{D}(\mathrm{D} / \mathrm{UW}-3 / \mathrm{CX})$ were purified and used to infect HeLa cells as described previously (Zhong et al., 2001). For some experiments, the culture media were supplemented with $50 \mu \mathrm{M}$ compound $\mathrm{C} 1$ (ChemBridge) or $10 \mu \mathrm{M}$ arylomycin C16 (kindly provided by The Scripps Research Institute; Roberts et al., 2007).

\section{Purification of CPAF from chlamydia-infected cultures for} determining the $\mathbf{N}$-terminal sequence. HeLa cells infected with $C$. trachomatis serovar D at an m.o.i. of 5 for $50 \mathrm{~h}$ were harvested from ten T-175 flasks and the cell pellets were lysed in $10 \mathrm{ml}$ MLB lysis buffer (25 mM HEPES, pH 7.5, $150 \mathrm{mM} \mathrm{NaCl}, 5 \%$ Igepal CA0630, $10 \mathrm{mM} \mathrm{MgCl} 2,5 \mathrm{mM}$ EDTA, $10 \%$ glycerol) with protease inhibitor cocktails containing $1 \mathrm{mM}$ PMSF, $20 \mu \mathrm{M}$ leupeptin, $1.6 \mu \mathrm{M}$ pepstatin A, $1.7 \mu \mathrm{g}$ aprotinin $\mathrm{ml}^{-1}$ (all from Sigma). The CPAF-containing supernatant was collected after centrifugation (20000 r.c.f., $1 \mathrm{~h}$ ) at $4{ }^{\circ} \mathrm{C}$ and incubated for $1 \mathrm{~h}$ at room temperature with the mouse monoclonal antibody (mAb) 100a (anti-CPAFc) that was covalently cross-linked to protein G Sepharose (GE Healthcare). The antibody cross-linking was carried out as follows: protein $\mathrm{G}$ beads were mixed with $\mathrm{mAb} 100 \mathrm{a}$ at $4{ }^{\circ} \mathrm{C}$ overnight. After washing away the free antibody molecules with $0.05 \%$ Tween 20 (Sigma) in PBS, the bound antibodies were covalently cross-linked to protein $\mathrm{G}$ by incubating in PBS containing $0.2 \mathrm{M}$ triethanolamine and $6.5 \mathrm{mg} \mathrm{ml}^{-1}$ of the crosslinker disuccinimidyl suberate (Pierce Biotechnology) at room temperature for $30 \mathrm{~min}$. Caution was taken not to over cross-link. Finally, free antibody molecules were washed away with $1 \mathrm{M}$ glycine in PBS (pH 3.0). After incubating with CPAF-containing supernatant, the bead complexes were thoroughly washed; the bound CPAF was eluted by using $2 \%$ SDS and was loaded onto a $12 \%$ SDS-PAGE gel. After electrophoresis, the resolved protein bands were blotted onto Sequi-Blot PVDF membrane (Bio-Rad). To visualize the CPAF protein bands, the membrane was briefly stained with Coomassie blue dye. The proCPAF band, migrating at $\sim 68 \mathrm{kDa}$, and the activated $\mathrm{N}$ terminal fragment CPAFn band, migrating at $\sim 29 \mathrm{kDa}$, were excised for N-terminal sequence analysis by using a commercial service provided by Alphalyse. A small portion of the precipitated CPAF sample was also subjected to Western blot analysis with mAb 54b to identify the N-terminus-containing CPAF bands (see below). Although a weak band migrating above the proCPAF, which may represent the precursor CPAF (with the signal peptide), was also subjected to $\mathrm{N}$-terminal sequencing analysis, no sequence information was obtained due to inadequate amounts of protein.

Bacterial strains and plasmid construction. E. coli $\mathrm{DH} 5 \alpha$ was purchased from Invitrogen. E. coli strains DR473 and DRS were reported previously (Marrichi et al., 2008) and strains JW3584-1, MC4100 and IQ85 were obtained from Yale University Genetic Stock Center (see http://cgsc.biology.yale.edu for detailed information). The DRS strain is derived from DR473 and is SecB-deficient; phoA is deleted from both of these strains (Marrichi et al., 2008). JW3584-1 is a derivative of MC4100 and is deficient in SecB. IQ85 is also a derivative of MC4100 but carries a temperature-sensitive mutation at SecY. JW3584-1, IQ85 and MC4100 maintain the endogenous phoA gene. The bacterial cells were grown in LB supplemented with antibiotics as follows: tetracycline for DR473 and DRS, spectinomycin and streptomycin for IQ85, kanamycin for JW3584-1 and streptomycin for MC4100 (each antibiotic at a final concentration of $50 \mu \mathrm{g}$ $\left.\mathrm{ml}^{-1}\right)$. In addition, ampicillin $\left(100 \mu \mathrm{g} \mathrm{ml}^{-1}\right)$ was added for culture of the strains transformed with pFLAG constructs.

The E. coli phoA gene with or without the signal peptide region was inserted into the XhoI/KpnI sites of pFLAG-CTC (Sigma) to create the recombinant plasmids pFLAG-PhoA (expressing full-length precursor PhoA) and pFLAG-'PhoA ('PhoA indicates a construct expressing mature PhoA without the signal peptide). To construct the plasmid pFLAG-CPAFss-' PhoA, a 93 bp DNA sequence coding for the CPAF signal peptide (M1-G31, designated CPAFss) was amplified from $C$. trachomatis serovar D and inserted into the XhoI/BamHI sites of the plasmid pBR322-'PhoA. The CPAFss-'PhoA was cloned from the pBR322 plasmid into pFLAG-CTC vector through the XhoI/KpnI sites. When the final three plasmids (pFLAG-PhoA, pFLAG-'PhoA and pFLAG-CPAFss-' $\mathrm{PhoA}$ ) were expressed in the above wild-type or mutant bacterial hosts, the FLAG tag in the pFLAG-CTC vector was added to the $\mathrm{C}$ terminus of PhoA.

5-Bromo-4-chloro-3-indolyl phosphate (BCIP) assay. The bacterial cells were grown in LB supplemented with the corresponding selective antibiotics at $37{ }^{\circ} \mathrm{C}$ overnight. The overnight cultures were streaked onto LB agar containing the same selective antibiotics and $50 \mu \mathrm{g} \mathrm{BCIP} \mathrm{ml} \mathrm{m}^{-1}$ (Sigma) and the plates were incubated at $30{ }^{\circ} \mathrm{C}$ for 2 days.

Western blot. The antibody-precipitated samples or bacterial samples were resolved by using SDS-PAGE and blotted onto nitrocellulose membranes to detect antigens with specific antibodies, as described previously (Zhong et al., 2001). The following primary antibodies were used: mouse mAb 54b against CPAFn (Zhong et al., 2001) at a final dilution of $1: 100$; mouse mAb M2 against FLAG tag (Sigma) at a final dilution of $1: 10000$; mouse $\mathrm{mAb}$ against maltose binding protein (MBP; Sigma); and rabbit polyclonal antibody against GroEL (Sigma) at a final dilution of $1: 10000$. The secondary antibodies were either goat anti-mouse or anti-rabbit IgG conjugated with horseradish peroxidase (HRP) at a final dilution of $1: 2000$. A standard enhanced chemiluminescent kit was used to visualize the antibody binding. The antibody-precipitated samples were prepared as described above. Both whole bacteria and bacterial cell fraction samples were prepared. The whole bacterial cells were fractionated into cytoplasmic and periplasmic fractions using a lysozyme-EDTA method, as described previously (Marrichi et al., 2008) with some modification. Briefly, a pellet from $10 \mathrm{ml}$ bacterial culture was washed with ice-cold PBS once and pelleted again by centrifugation at 
6000 r.c.f. for $10 \mathrm{~min}$ at $4{ }^{\circ} \mathrm{C}$. The pelleted bacterial cells were resuspended in $0.5 \mathrm{ml}$ periplasting buffer containing $20 \mathrm{mM}$ Tris/ $\mathrm{HCl}$ (pH 7.5), $20 \%$ sucrose (EMD Chemicals), $1 \mathrm{mM}$ EDTA (Sigma), $3 \mathrm{mg}$ lysozyme $\mathrm{ml}^{-1}$ (MP biomedicals). After incubating on ice for $5 \mathrm{~min}, 0.5 \mathrm{ml}$ ice-cold distilled water was added to the suspension and mixed by pipetting up and down. After incubating on ice for another $5 \mathrm{~min}$, the mixture was pelleted by centrifugation at $12000 \mathrm{~g}$ for $2 \mathrm{~min}$ at $4{ }^{\circ} \mathrm{C}$. The periplasmic fraction in the supernatant was collected to a new tube while the cytoplasmic proteins in the remaining pellet were resuspended in $1 \mathrm{ml}$ periplasting buffer. The quality of the fractionated samples was monitored by immunodetection of both the periplasmic protein MBP and the cytoplasmic protein GroEL.

Immunofluorescence assay. Chlamydia-infected cells grown on coverslips were processed for antibody labelling to visualize specific antigens using an immunofluorescence assay as described previously (Zhong et al., 2001). Briefly, HeLa cells were infected with L2 organisms at an m.o.i. of 0.5. Eight hours after infection, the culture medium was replaced with fresh medium containing DMSO, C1 compound or arylomycin $\mathrm{C} 16$. At $36 \mathrm{~h}$ post-infection, the culture samples were fixed with $2 \%$ paraformaldehyde (Sigma) for $30 \mathrm{~min}$ at room temperature and permeabilized with $2 \%$ saponin (Sigma) for $30 \mathrm{~min}$ at room temperature. After blocking with DMEM/10\% FBS for $1 \mathrm{~h}$ at room temperature, the following primary antibodies were used: a rabbit antibody against chlamydial EB organisms (unpublished data) at a dilution of $1: 2000$; a mouse $\mathrm{mAb}$ against IncA (clone no. CB9, mIgG1; unpublished data) at a final dilution of $1: 50$; a mouse mAb against CPAF (clone no. IL4A6, mIgG2b; unpublished data) at a final dilution of $1: 50$. The primary antibody labelling was visualized with the secondary antibodies goat anti-mouse IgG2b conjugated with $\mathrm{Cy} 3$ (red), goat anti-mouse IgG1 conjugated with Cy2 (green) and goat anti-rabbit IgG conjugated with Cy5 (blue). The triple-labelled samples were observed under a fluorescence microscope (AX-70, Olympus) equipped with a Hamamatsu CCD camera and the Simple PCI imaging software. The images were acquired one colour at a time and overlaid with Adobe photoshop software.

\section{RESULTS}

\section{The CPAF $\mathbf{N}$-terminal signal peptide is cleaved during chlamydial infection}

We analysed the amino acid sequence of CPAF encoded by C. trachomatis serovar D for potential secretion signal peptides using the program SignalP version 3.0 with neural network (NN) and hidden Markov model (HMM) algorithms (www.expasy.ch). Both NN and HMM algorithms predict an N-terminal signal peptide in CPAF but with different cleavage sites. NN predicts a cleavage between His26 and Ser27 while HMM predicts the cleavage site between Gly31 and Glu32 (Fig. 1a). To test whether the predicted N-terminal signal peptide is indeed cleaved during chlamydial infection and to determine the cleavage site, we analysed the N-terminal sequence of CPAF purified from chlamydia-infected cells (Fig. 1b). An anti-CPAFc $\mathrm{mAb}$ was used to affinity purify CPAF and the processed fragments from chlamydia-infected cells. Bands representing the precursor and mature full-length $\mathrm{CPAF}$ and $\mathrm{CPAFn}$ were excised from the PVDF membrane for $\mathrm{N}$-terminal sequencing. The precursor CPAF band generated no amino acid information due to insufficient amounts of protein or to N-terminal blocking. However, both the mature fulllength CPAF and activated CPAFn bands produced the six amino acids ESLVCK, which matches residues 32-37 of $\mathrm{CPAF}$, indicating that a sequence covering residues 1-31 was cleaved from CPAF; this confirms the results predicted by the HMM algorithm. The cleavage site of Gly31 and Glu32 is conserved among serovars of C. trachomatis and Chlamydia muridarum species but is absent in CPAF from other chlamydial species, suggesting that an alternative cleavage site may be used by other chlamydial species.

\section{The putative $\mathbf{N}$-terminal signal peptide of CPAF is able to direct export of PhoA into the bacterial periplasmic space}

Due to a lack of genetic tools for manipulating the chlamydial genome, we tested the functionality of the putative CPAF signal peptide using a bacterium-based phoA gene fusion system. This assay system exploits two characteristics of PhoA: the enzyme is only active after translocation into the bacterial periplasm, and the phosphatase activity can be conveniently monitored with the chromogenic substrate BCIP (Marrichi et al., 2008). DNA (a)

N-MGFWRTSIMKMNRIWLLLLTFSSAIH ${ }_{26} \mathrm{SPVQG}_{31}$ ESLVCKNALQDL...

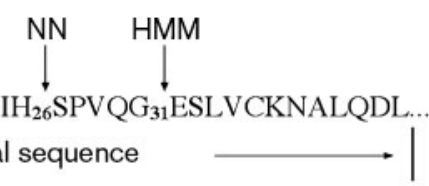

(b)

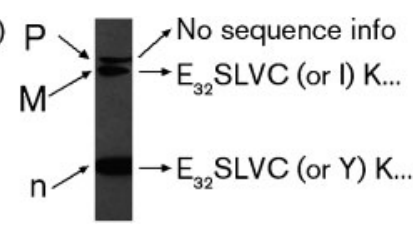

Fig. 1. Prediction of CPAF signal peptide and N-terminal sequencing of CPAF. (a) The SignalP 3.0 program with both the NN and HMM algorithms (www.expasy.ch) was used to analyse the precursor CPAF sequence from C. trachomatis serovar D (http:// stdgen.northwestern.edu/). The NN algorithm predicts a signal peptide covering a region from the methionine residue at position $\mathrm{M} 1$ to the histidine residue at position $26(\mathrm{H} 26)$ while the HMM-predicted signal peptide extends to residue G31 (M1-G31). (b) Endogenous CPAF was immunoprecipitated from chlamydia-infected cell lysates using the mAb $100 a ;>95 \%$ of the precipitate was resolved by using SDS-PAGE and was transferred onto a PVDF membrane for N-terminal sequencing using the Edman degradation method. The first six residues derived from the corresponding bands are listed to the right of the gel. The remaining $<5 \%$ of the precipitate was also resolved by using SDS-PAGE and analysed via Western blot detection of the N-terminuscontaining CPAF bands with mAb 54b. P, Precursor CPAF; M, mature full-length processed CPAF; n, processed CPAFn. 
coding for the CPAF $\mathrm{N}$-terminal signal sequence covering residues M1-G31 (designated CPAFss) was fused to the DNA sequence coding for mature PhoA (designated 'PhoA). The fusion construct was expressed in pFLAGCTC which adds a FLAG epitope to the $\mathrm{C}$ terminus of 'PhoA. The mature 'PhoA alone construct was used as a negative control while the precursor full-length PhoA (with its native $\mathrm{N}$-terminal signal peptide) served as a positive control. In the presence of BCIP, bacteria expressing either the precursor PhoA or the CPAFss-'PhoA fusion constructs turned blue, whereas bacteria expressing the mature PhoA alone ('PhoA) remained white, indicating that both the native PhoA signal peptide and CPAF signal peptides directed the translocation of PhoA into the periplasm (Fig. 2a). We used Western blot analysis to monitor the distribution of PhoA protein in periplasmic and cytosolic fractions of the bacteria expressing these constructs (Fig. 2b). Mature PhoA was detected in the periplasm of bacteria expressing either the precursor PhoA or CPAFss'PhoA fusion constructs, while mature PhoA was only detected in the cytoplasm of the bacteria expressing the leaderless PhoA. Thus, the CPAF N-terminal signal peptide is sufficient for directing PhoA across the bacterial inner membrane.

\section{The CPAF signal peptide-directed translocation of PhoA requires the Sec-dependent pathway}

To further evaluate the role of the Sec-dependent pathway in CPAF signal peptide-directed translocation of PhoA, export of mature PhoA encoded by the CPAFss-'PhoA fusion construct was compared between bacteria with or without competent $\sec B$ (Fig. 3) or $\sec Y$ (Fig. 4). When the CPAFss-'PhoA fusion construct was expressed in the bacterial strain DR473 that expresses functional SecB, the colonies turned blue on BCIP plates. In contrast, the DRS strain that lacks SecB appeared as white colonies when the same fusion construct was expressed (Fig. 3a), demonstrating that CPAFss can direct mature PhoA to access the periplasm in SecB-competent but not SecB-deficient bacteria. This difference in PhoA export observed in SecB-competent and SecB-deficient bacteria was confirmed using a combination of bacterial cell fractionation and Western blot analysis (Fig. 3b). Mature PhoA was predominantly detected in the periplasm of SecB-competent bacteria while the precursor PhoA remained in the cytosolic fraction of the SecB-deficient bacteria. This was confirmed by data from another pair of SecB-competent and -deficient bacteria that carry the endogenous phoA gene (Fig. 3b, ii and iv). Native PhoA secretion is known to be independent of SecB (Marrichi et al., 2008); however, the CPAF leader-peptide-directed export of PhoA was observed to be dependent on SecB, suggesting that it is the CPAF leader peptide that renders the SecB-dependence. To determine whether the CPAF signal peptide-directed protein export also required SecY, we compared the translocation of the mature PhoA encoded by the CPAFss-'PhoA fusion construct between MC4100, which (a)

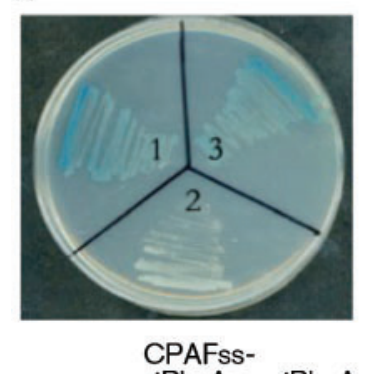

(b) $\frac{\text { 'PhoA }}{\text { per cyt }} \frac{\text { 'PhoA }}{\text { per cyt }} \frac{\text { PhoA }}{\text { per cyt }}$

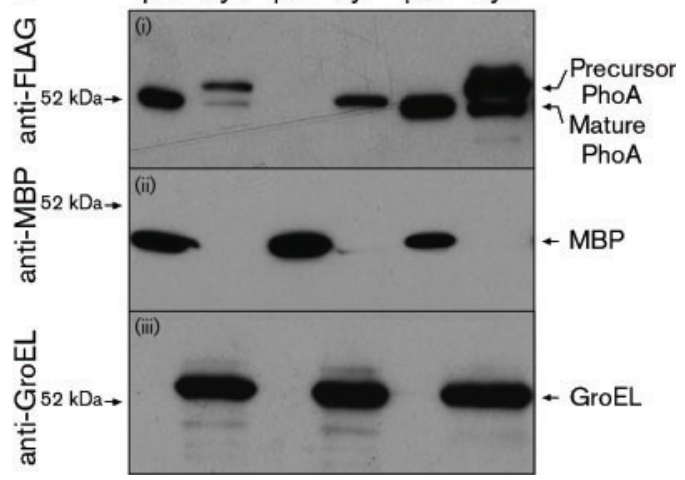

Fig. 2. The putative $\mathrm{N}$-terminal signal peptide of $\mathrm{CPAF}$ is able to direct translocation of mature PhoA into the bacterial periplasmic space. (a) Constructs encoding CPAF N-terminal peptide-mature PhoA fusion protein (CPAFss-'PhoA; 1 ), mature PhoA (without a signal peptide, 'PhoA; 2 ) or full-length $\mathrm{PhoA}$ (with its intrinsic signal peptide; 3) were expressed in $E$. coli $\mathrm{DH} 5 \alpha$ cells lacking endogenous PhoA. The transformed bacteria were incubated at $30{ }^{\circ} \mathrm{C}$ for $48 \mathrm{~h}$ on agar plates supplemented with BCIP. PhoA translocated into the periplasmic space is able to catalyse BCIP to produce a blue colour. Note that expression of the constructs encoding PhoA carrying either the native or CPAF signal peptide led to blue colonies, while the colonies expressing the mature PhoA alone remained white. (b) Bacterial transformants expressing the same three constructs were also fractionated into periplasmic (per) and cytosolic (cyt) fractions; these were separated by SDS-PAGE, transferred to a PVDF membrane by Western blot and detected with antibodies against a FLAG tag (anti-FLAG, i), MBP (anti-MBP, ii) and GroEL (anti-GroEL, iii). Note that mature PhoA was secreted into the periplasm of bacteria expressing either the full-length $P h o A$ construct or CPAFss-'PhoA construct, while mature PhoA stayed in the cytoplasm of the bacteria expressing the mature PhoA alone construct.

carries wild-type $\sec Y$, and IQ85, which expresses a temperature-sensitive $\sec Y$ allele (Fig. 4). At the permissive temperature $\left(30{ }^{\circ} \mathrm{C}\right)$, both bacterial strains efficiently processed the precursor protein and exported mature PhoA into the periplasmic space. However, at the restrictive temperature $\left(42{ }^{\circ} \mathrm{C}\right), \mathrm{MC} 4100$ cells were able to process precursor PhoA into mature PhoA and to export mature PhoA into the periplasm, while IQ85 cells failed to do so, suggesting that the CPAF signal peptide-assisted translocation of mature PhoA is also dependent on SecY. 


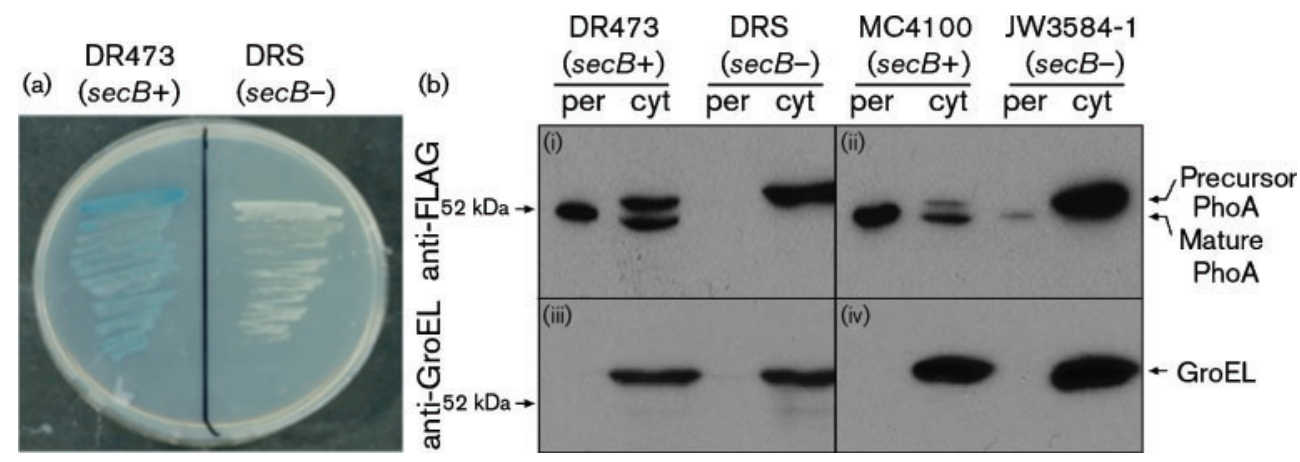

Fig. 3. The CPAF signal-peptide-directed translocation of mature PhoA is dependent on $\mathrm{Sec} B$. (a) The construct coding for the CPAF signal peptide-mature PhoA fusion protein (CPAFss-'PhoA-FLAG) was transformed into a mutant bacterial strain deficient in $\sec B$ (DRS) or its wild-type control strain (DR473). The transformed bacteria were cultured for $48 \mathrm{~h}$ at $30{ }^{\circ} \mathrm{C}$ on agar plates supplemented with BCIP. Note that SecB-deficient bacteria remained white while the wild-type bacteria turned blue. (b) The parallel bacterial cultures were harvested for fractionation. The presence of PhoA in both the periplasmic (per) and cytosolic (cyt) fractions was analysed by Western blot as described in the legend to Fig. 2(b). The fusion construct was also tested in an independent SecB-deficient mutant strain JW3584-1 and its corresponding control, wild-type MC4100. Note that both SecB-deficient bacterial strains failed to support PhoA export into the periplasm.

These data clearly show that both $\operatorname{SecB}$ and SecY are required for the functionality of the CPAF $\mathrm{N}$-terminal signal peptide.

\section{CPAF secretion during chlamydial infection requires the Sec-dependent but not the type III secretion pathway}

Having demonstrated the functionality of the CPAF Nterminal secretion signal peptide in a heterologous system, we further tested whether CPAF secretion in chlamydiainfected cells is also dependent on the general secretory pathway. To bypass the difficulty in genetic manipulation of the chlamydial genome, we used the compound arylomycin C16, a lipopeptide-based inhibitor specifically targeting prokaryotic signal peptidase I (Luo et al., 2009; Paetzel et al., 2004), to block the chlamydial general secretory pathway (Fig. 5). Since inhibition of the general secretory pathway also leads to inhibition of organism growth, chlamydial infection was allowed for $8 \mathrm{~h}$ prior to the drug treatment and the appropriate drug concentration was used to permit sufficient levels of protein biosynthesis for monitoring translocation. We found that arylomycin $\mathrm{C} 16$ at $10 \mu \mathrm{M}$ completely blocked CPAF secretion into

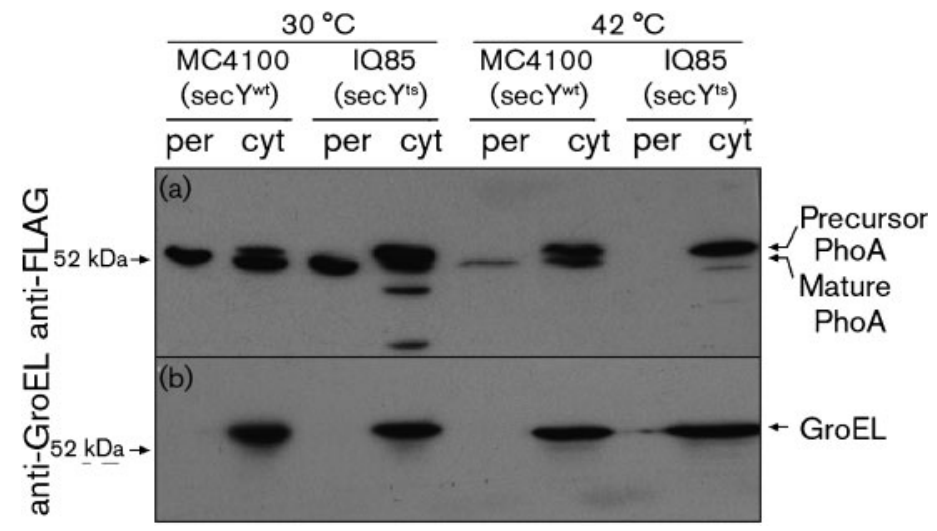

Fig. 4. The CPAF signal-peptide-directed export of mature PhoA also requires SecY. The construct coding for the CPAF signal peptidemature PhoA fusion protein (CPAFss-'PhoAFLAG) was transformed into bacterial strains that carry either a wild-type $\sec Y\left(\sec Y^{w t}\right)$ or a temperature-sensitive mutant $\sec Y$ allele $\left(\mathrm{sec} \mathrm{Y}^{\mathrm{ts}}\right)$. The overnight culture incubated at $30{ }^{\circ} \mathrm{C}$ was diluted into fresh medium to reach $\mathrm{OD}_{600}=0.1$. Half of the culture was grown at $30{ }^{\circ} \mathrm{C}$ and the other half was grown at $42{ }^{\circ} \mathrm{C}$ for another $4 \mathrm{~h}$. The parallel cultures were harvested for fractionation. The presence of PhoA in both the periplasmic (per) and cytosolic (cyt) fractions was analysed by Western blot as described in the legend to Fig. 2(b). Note that at the permissive temperature, bacterial strains carrying either sec $Y^{w t}$ or sec $Y^{\text {ts }}$ exported mature PhoA into the periplasm while at the restrictive temperature, only the $\sec Y^{w t}$ strain but not the sec $Y^{\text {ts }}$ strain exported mature PhoA into the periplasm. 


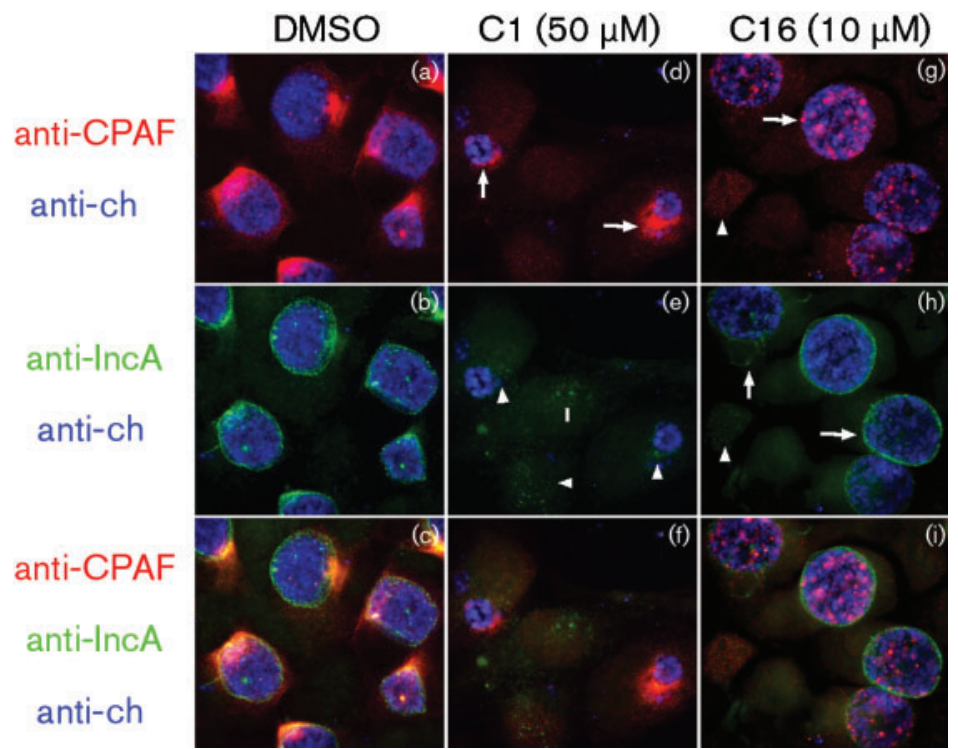

Fig. 5. CPAF secretion into the cytosol of chlamydia-infected cells is inhibited by arylomycin $\mathrm{C} 16$, an inhibitor targeting prokaryotic signal peptidase I. HeLa monolayers were infected with C. trachomatis L2 at an m.o.i. of 0.5 for $8 \mathrm{~h}$. The cultures were treated with DMSO (a-c), $50 \mu \mathrm{M} \mathrm{C1}$ compound (d-f; an inhibitor targeting the Gram-negative bacterial type III secretion system) or $10 \mu \mathrm{M} \mathrm{C} 16$ (g-i; an inhibitor targeting the signal peptidase I). Twenty-four hours after treatment, the cultures were processed for the immunofluorescence assay. The processed monolayers were immuno-labelled with antibodies against chlamydia (blue), IncA (green) and CPAF (red). Note that $C 1$ selectively inhibits secretion of IncA but not CPAF, while C16 blocks secretion of CPAF but not IncA. White arrows indicate specific labelling while white arrowheads represent possible non-specific signals. host cells when added $8 \mathrm{~h}$ after infection (Fig. 5g-i). Importantly, this treatment did not significantly affect the inclusion membrane localization of IncA. This is because secretion of IncA into the inclusion membrane is known to depend on the type III secretion system and not the general secretory pathway. Indeed, treatment of the chlamydial culture with the $\mathrm{C} 1$ compound, a small molecule inhibitor known to block type III secretion in both chlamydia and other Gram-negative bacteria, led to complete blockage of IncA localization into the inclusion membrane without affecting the secretion of CPAF into host cell cytosol (Fig. 5d-f). Thus, we have demonstrated that CPAF secretion requires a functional signal peptidase but is independent of the type III secretion system.

\section{DISCUSSION}

CPAF was the first identified secreted protein from chlamydial organisms and has been characterized extensively, including the recently resolved crystal structure (Dong et al., 2004a, b, c, 2005; Huang et al., 2008; Zhong et al., 1999, 2000, 2001). However, its secretion pathway has remained elusive. Although a putative secretion signal peptide has been predicted at the $\mathrm{N}$ terminus of $\mathrm{CPAF}$, no experimental evidence has ever been presented to identify the CPAF secretion pathway(s). Here, we have presented experimental data that convincingly demonstrate that CPAF secretion requires the Sec-dependent pathway. First, the predicted putative N-terminal sequence covering residues M1-G31 is removed from CPAF in chlamydiainfected cells, which is consistent with the observation that CPAF without the signal peptide maintains the tertiary structure required for its activity (Huang et al., 2008). Second, the CPAF secretion signal peptide is able to direct translocation of the mature bacterial phosphatase PhoA into the bacterial periplasmic space when expressed in a fusion construct via a transgene, indicating that the CPAF secretion signal peptide is recognized by bacterial secretion chaperones and translocons and is cleavable by bacterial signal peptidases. Third, the CPAF signal peptide-assisted translocation of PhoA is dependent on both $\mathrm{SecB}$ and $\mathrm{SecY}$, further confirming that both the chaperone and the Sec translocon are required for the CPAF signal peptide to exert its function. Finally, and most importantly, CPAF secretion in chlamydia-infected cells can be blocked by a small molecule inhibitor arylomycin C16 that specifically inhibits prokaryotic signal peptidases but cannot be blocked by the $\mathrm{C} 1$ compound that targets the type III secretion system, demonstrating for the first time that chlamydial secretion of CPAF is dependent on a functional signal peptidase. The chlamydial genome does encode a putative type I signal peptidase (designated CT020; http:// stdgen.northwestern.edu/). The finding that CPAF secretion is not inhibited by the $\mathrm{C} 1$ compound is consistent with previous observations (Betts et al., 2009; Jorgensen \& Valdivia, 2008) and is also in agreement with the observation that CPAF either full-length or in fragments cannot be secreted by the type III secretion system of Yersinia endotuberculosis (our unpublished observations). Thus, we can conclude that a Sec-dependent, but not a type III secretion pathway, is required for CPAF secretion.

Previous characterization of CPAF has revealed that CPAF possesses many common features of bacterial exotoxins. For example, many exotoxins are processed and some possess proteolytic activity targeting host proteins to altering host cell signalling (Bhatnagar \& Batra, 2001). CPAF is a serine protease and can degrade various host proteins for manipulating host cell signalling pathways (Zhong et al., 1999, 2000, 2001). CPAF is also processed into two fragments (Dong et al., 2004a). In the current study, we have revealed that CPAF, like many bacterial 
exotoxins (Lee \& Schneewind, 2001), is secreted out of chlamydial organisms via the general secretory pathway. The next question is how the periplasmic CPAF is translocated out of chlamydial organisms and further out of chlamydial inclusions into host cells. Some bacterial toxins follow the classical type II secretion pathway by passing through an outer membrane pore created by the oligomerization of the GspD to exit the bacterial cells while others can be released by outer membrane vesicles (OMVs) (Lee et al., 2008; Mashburn-Warren et al., 2008; Mashburn-Warren \& Whiteley, 2006). The OMV-trapped toxins can travel far to reach their target cells (Bomberger et al., 2009). However, it is not known how CPAF passes through the outer membrane of chlamydial organisms. Although the chlamydial genome does encode a GspD homologue, it is unlikely that CPAF exits the chlamydial organisms through the outer membrane pore. This is because releasing free CPAF molecules into the lumen of the inclusion may make it difficult for CPAF to subsequently translocate into host cells. We propose that the periplasmic CPAF may use an outer membrane budding mechanism to exit the chlamydial organisms. In this way, CPAF molecules are sequestered within the budding vesicles when the OMVs are still in the lumen of the chlamydial inclusions. Free CPAF molecules can then be released into host cells when the CPAF-containing OMVs fuse with the inclusion membrane or the entire OMVs bud off the inclusions into host cells. This hypothesis is supported by the observations that the chlamydial RB outer membrane can undergo extensive vesiculation under certain culture conditions (Matsumoto \& Manire, 1970), chlamydial organism-free vesicles are detected inside the lumen of chlamydial inclusions (Jorgensen \& Valdivia, 2008) and the release of vesicles from chlamydial inclusions can be induced (Giles et al., 2006). Efforts are underway to dissect the precise pathways required for $\mathrm{CPAF}$ to enter host cell cytoplasmic space for targeting host cell proteins.

Regardless of the precise mechanisms by which CPAF exits the periplasmic space and enters into the host cell cytosol, the current finding has highlighted the importance of the general secretory pathway in chlamydial pathogenesis. The type III secretion system has been found to secrete many chlamydial substrates/effectors into the inclusion membrane (Valdivia, 2008) and host cells (Clifton et al., 2004; Valdivia, 2008) and has been extensively studied (Betts et al., 2009). We have demonstrated that the type II secretion pathway can also participate in the secretion of chlamydial effectors into the host cell cytoplasm as suggested previously (Jorgensen \& Valdivia, 2008; Valdivia, 2008). In addition to CPAF, other putative effectors are also predicted to carry $\mathrm{N}$-terminal signal peptides, including Tsp (CT441) that is known to cleave the host cell protein NF- $\kappa \mathrm{B}$ (Lad et al., 2007). It appears that searching for chlamydial effectors that can be secreted via the Sec-dependent pathway may represent another productive approach for unravelling new mechanisms of chlamydial interactions with host cells.

\section{ACKNOWLEDGEMENTS}

This work was supported in part by grants (to G. Z.) from the US National Institutes of Health.

\section{REFERENCES}

Baba, T., Jacq, A., Brickman, E., Beckwith, J., Taura, T., Ueguchi, C., Akiyama, Y. \& Ito, K. (1990). Characterization of cold-sensitive secY mutants of Escherichia coli. J Bacteriol 172, 7005-7010.

Betts, H. J., Wolf, K. \& Fields, K. A. (2009). Effector protein modulation of host cells: examples in the Chlamydia spp. arsenal. Curr Opin Microbiol 12, 81-87.

Bhatnagar, R. \& Batra, S. (2001). Anthrax toxin. Crit Rev Microbiol 27, 167-200.

Bomberger, J. M., Maceachran, D. P., Coutermarsh, B. A., Ye, S., O'Toole, G. A. \& Stanton, B. A. (2009). Long-distance delivery of bacterial virulence factors by Pseudomonas aeruginosa outer membrane vesicles. PLoS Pathog 5, e1000382.

Campbell, L. A. \& Kuo, C. C. (2004). Chlamydia pneumoniae - an infectious risk factor for atherosclerosis? Nat Rev Microbiol 2, 23-32.

Clifton, D. R., Fields, K. A., Grieshaber, S. S., Dooley, C. A., Fischer, E. R., Mead, D. J., Carabeo, R. A. \& Hackstadt, T. (2004). A chlamydial type III translocated protein is tyrosine-phosphorylated at the site of entry and associated with recruitment of actin. Proc Natl Acad Sci U S A 101, 10166-10171.

Collinson, I. (2005). The structure of the bacterial protein translocation complex SecYEG. Biochem Soc Trans 33, 1225-1230.

Dalbey, R. E. (1991). Leader peptidase. Mol Microbiol 5, 2855-2860.

Dong, F., Pirbhai, M., Zhong, Y. \& Zhong, G. (2004a). Cleavagedependent activation of a chlamydia-secreted protease. Mol Microbiol 52, 1487-1494.

Dong, F., Sharma, J., Xiao, Y., Zhong, Y. \& Zhong, G. (2004b). Intramolecular dimerization is required for the chlamydia-secreted protease CPAF to degrade host transcriptional factors. Infect Immun 72, 3869-3875.

Dong, F., Su, H., Huang, Y., Zhong, Y. \& Zhong, G. (2004c). Cleavage of host keratin 8 by a chlamydia-secreted protease. Infect Immun 72, 3863-3868.

Dong, F., Zhong, Y., Arulanandam, B. \& Zhong, G. (2005). Production of a proteolytically active protein, chlamydial protease/ proteasome-like activity factor, by five different chlamydia species. Infect Immun 73, 1868-1872.

Dong, F., Flores, R., Chen, D., Luo, J., Zhong, Y., Wu, Z. \& Zhong, G. (2006). Localization of the hypothetical protein Cpn0797 in the cytoplasm of Chlamydia pneumoniae-infected host cells. Infect Immun 74, 6479-6486.

Driessen, A. J. \& Nouwen, N. (2008). Protein translocation across the bacterial cytoplasmic membrane. Annu Rev Biochem 77, 643-667.

Economou, A. (1999). Following the leader: bacterial protein export through the Sec pathway. Trends Microbiol 7, 315-320.

Eissenberg, L. G., Wyrick, P. B., Davis, C. H. \& Rumpp, J. W. (1983). Chlamydia psittaci elementary body envelopes: ingestion and inhibition of phagolysosome fusion. Infect Immun 40, 741-751.

Fan, T., Lu, H., Hu, H., Shi, L., McClarty, G. A., Nance, D. M., Greenberg, A. H. \& Zhong, G. (1998). Inhibition of apoptosis in chlamydia-infected cells: blockade of mitochondrial cytochrome c release and caspase activation. J Exp Med 187, 487-496.

Fan, P., Dong, F., Huang, Y. \& Zhong, G. (2002). Chlamydia pneumoniae secretion of a protease-like activity factor for 
degrading host cell transcription factors required for major histocompatibility complex antigen expression. Infect Immun 70, 345-349.

Fields, K. A. \& Hackstadt, T. (2000). Evidence for the secretion of Chlamydia trachomatis CopN by a type III secretion mechanism. Mol Microbiol 38, 1048-1060.

Fields, K. A., Mead, D. J., Dooley, C. A. \& Hackstadt, T. (2003). Chlamydia trachomatis type III secretion: evidence for a functional apparatus during early-cycle development. Mol Microbiol 48, 671683.

Francetic, O. \& Kumamoto, C. A. (1996). Escherichia coli SecB stimulates export without maintaining export competence of ribosebinding protein signal sequence mutants. J Bacteriol 178, 59545959.

Giles, D. K., Whittimore, J. D., LaRue, R. W., Raulston, J. E. \& Wyrick, P. B. (2006). Ultrastructural analysis of chlamydial antigen-containing vesicles everting from the Chlamydia trachomatis inclusion. Microbes Infect 8, 1579-1591.

Greene, W., Xiao, Y., Huang, Y., McClarty, G. \& Zhong, G. (2004). Chlamydia-infected cells continue to undergo mitosis and resist induction of apoptosis. Infect Immun 72, 451-460.

Hackstadt, T., Fischer, E. R., Scidmore, M. A., Rockey, D. D. \& Heinzen, R. A. (1997). Origins and functions of the chlamydial inclusion. Trends Microbiol 5, 288-293.

Hefty, P. S. \& Stephens, R. S. (2007). Chlamydial type III secretion system is encoded on ten operons preceded by sigma 70-like promoter elements. J Bacteriol 189, 198-206.

Hobolt-Pedersen, A. S., Christiansen, G., Timmerman, E., Gevaert, K. \& Birkelund, S. (2009). Identification of Chlamydia trachomatis CT621, a protein delivered through the type III secretion system to the host cell cytoplasm and nucleus. FEMS Immunol Med Microbiol 57, 4658.

Huang, Z., Feng, Y., Chen, D., Wu, X., Huang, S., Wang, X., Xiao, X., Li, W., Huang, N. \& other authors (2008). Structural basis for activation and inhibition of the secreted chlamydia protease CPAF. Cell Host Microbe 4, 529-542.

Hybiske, K. \& Stephens, R. S. (2007). Mechanisms of host cell exit by the intracellular bacterium Chlamydia. Proc Natl Acad Sci U S A 104, 11430-11435.

Jorgensen, I. \& Valdivia, R. H. (2008). Pmp-like proteins Pls1 and Pls2 are secreted into the lumen of the Chlamydia trachomatis inclusion. Infect Immun 76, 3940-3950.

Karunakaran, K. P., Noguchi, Y., Read, T. D., Cherkasov, A., Kwee, J., Shen, C., Nelson, C. C. \& Brunham, R. C. (2003). Molecular analysis of the multiple GroEL proteins of Chlamydiae. J Bacteriol 185, 19581966

Kumar, Y. \& Valdivia, R. H. (2008). Actin and intermediate filaments stabilize the Chlamydia trachomatis vacuole by forming dynamic structural scaffolds. Cell Host Microbe 4, 159-169.

Lad, S. P., Li, J., da Silva Correia, J., Pan, Q., Gadwal, S., Ulevitch, R. J. \& Li, E. (2007). Cleavage of p65/RelA of the NF- $\kappa$ B pathway by Chlamydia. Proc Natl Acad Sci U S A 104, 2933-2938.

Lee, V. T. \& Schneewind, O. (2001). Protein secretion and the pathogenesis of bacterial infections. Genes Dev 15, 1725-1752.

Lee, E. Y., Choi, D. S., Kim, K. P. \& Gho, Y. S. (2008). Proteomics in Gram-negative bacterial outer membrane vesicles. Mass Spectrom Rev 27, 535-555.

Li, Z., Chen, C., Chen, D., Wu, Y., Zhong, Y. \& Zhong, G. (2008a). Characterization of fifty putative inclusion membrane proteins encoded in the Chlamydia trachomatis genome. Infect Immun 76, 2746-2757.
Li, Z., Chen, D., Zhong, Y., Wang, S. \& Zhong, G. (2008b). The chlamydial plasmid-encoded protein pgp3 is secreted into the cytosol of Chlamydia-infected cells. Infect Immun 76, 3415-3428.

Luo, C., Roussel, P., Dreier, J., Page, M. G. \& Paetzel, M. (2009). Crystallographic analysis of bacterial signal peptidase in ternary complex with arylomycin A2 and a $\beta$-sultam inhibitor. Biochemistry 48, 8976-8984.

Mabey, D. (2008). Trachoma: recent developments. Adv Exp Med Biol 609, 98-107.

Mårdh, P. A. (2004). Tubal factor infertility, with special regard to chlamydial salpingitis. Curr Opin Infect Dis 17, 49-52.

Marrichi, M., Camacho, L., Russell, D. G. \& DeLisa, M. P. (2008). Genetic toggling of alkaline phosphatase folding reveals signal peptides for all major modes of transport across the inner membrane of bacteria. J Biol Chem 283, 35223-35235.

Mashburn-Warren, L. M. \& Whiteley, M. (2006). Special delivery: vesicle trafficking in prokaryotes. Mol Microbiol 61, 839-846.

Mashburn-Warren, L., McLean, R. J. \& Whiteley, M. (2008). Gramnegative outer membrane vesicles: beyond the cell surface. Geobiology 6, 214-219.

Matsumoto, A. \& Manire, G. P. (1970). Electron microscopic observations on the effects of penicillin on the morphology of Chlamydia psittaci. J Bacteriol 101, 278-285.

Paetzel, M., Karla, A., Strynadka, N. C. \& Dalbey, R. E. (2002). Signal peptidases. Chem Rev 102, 4549-4580.

Paetzel, M., Goodall, J. J., Kania, M., Dalbey, R. E. \& Page, M. G. (2004). Crystallographic and biophysical analysis of a bacterial signal peptidase in complex with a lipopeptide-based inhibitor. J Biol Chem 279, 30781-30790.

Pirbhai, M., Dong, F., Zhong, Y., Pan, K. Z. \& Zhong, G. (2006). The secreted protease factor CPAF is responsible for degrading proapoptotic BH3-only proteins in Chlamydia trachomatis-infected cells. J Biol Chem 281, 31495-31501.

Pugsley, A. P., d'Enfert, C., Reyss, I. \& Kornacker, M. G. (1990). Genetics of extracellular protein secretion by Gram-negative bacteria. Annu Rev Genet 24, 67-90.

Pugsley, A. P., Francetic, O., Driessen, A. J. \& de Lorenzo, V. (2004). Getting out: protein traffic in prokaryotes. Mol Microbiol 52, 3-11.

Roberts, T. C., Smith, P. A., Cirz, R. T. \& Romesberg, F. E. (2007). Structural and initial biological analysis of synthetic arylomycin A2. J Am Chem Soc 129, 15830-15838.

Rockey, D. D., Heinzen, R. A. \& Hackstadt, T. (1995). Cloning and characterization of a Chlamydia psittaci gene coding for a protein localized in the inclusion membrane of infected cells. Mol Microbiol 15, 617-626.

Rzomp, K. A., Moorhead, A. R. \& Scidmore, M. A. (2006). The GTPase Rab4 interacts with Chlamydia trachomatis inclusion membrane protein CT229. Infect Immun 74, 5362-5373.

Saier, M. H., Jr (2006). Protein secretion and membrane insertion systems in gram-negative bacteria. J Membr Biol 214, 75-90.

Spaeth, K. E., Chen, Y. S. \& Valdivia, R. H. (2009). The Chlamydia type III secretion system C-ring engages a chaperone-effector protein complex. PLoS Pathog 5, e1000579.

Stathopoulos, C., Hendrixson, D. R., Thanassi, D. G., Hultgren, S. J., St Geme, J. W., III \& Curtiss, R., III (2000). Secretion of virulence determinants by the general secretory pathway in Gram-negative pathogens: an evolving story. Microbes Infect 2, 1061-1072.

Stephens, R. S. (2003). The cellular paradigm of chlamydial pathogenesis. Trends Microbiol 11, 44-51.

Su, H., McClarty, G., Dong, F., Hatch, G. M., Pan, Z. K. \& Zhong, G. (2004). Activation of Raf/MEK/ERK/cPLA2 signaling pathway is 
essential for chlamydial acquisition of host glycerophospholipids. J Biol Chem 279, 9409-9416.

Tuteja, R. (2005). Type I signal peptidase: an overview. Arch Biochem Biophys 441, 107-111.

Valdivia, R. H. (2008). Chlamydia effector proteins and new insights into chlamydial cellular microbiology. Curr Opin Microbiol 11, 53-59.

Vandahl, B. B., Stensballe, A., Roepstorff, P., Christiansen, G. \& Birkelund, S. (2005). Secretion of Cpn0796 from Chlamydia pneumoniae into the host cell cytoplasm by an autotransporter mechanism. Cell Microbiol 7, 825-836.

Xiao, Y., Zhong, Y., Su, H., Zhou, Z., Chiao, P. \& Zhong, G. (2005). $\mathrm{NF}-\kappa \mathrm{B}$ activation is not required for Chlamydia trachomatis inhibition of host epithelial cell apoptosis. J Immunol 174, 17011708.

Zhong, G. (2009). Killing me softly: chlamydial use of proteolysis for evading host defenses. Trends Microbiol 17, 467-474.
Zhong, G., Fan, T. \& Liu, L. (1999). Chlamydia inhibits interferon gamma-inducible major histocompatibility complex class II expression by degradation of upstream stimulatory factor 1. J Exp Med 189, 1931-1938.

Zhong, G., Liu, L., Fan, T., Fan, P. \& Ji, H. (2000). Degradation of transcription factor RFX5 during the inhibition of both constitutive and interferon gamma-inducible major histocompatibility complex class I expression in chlamydia-infected cells. J Exp Med 191, 15251534.

Zhong, G., Fan, P., Ji, H., Dong, F. \& Huang, Y. (2001). Identification of a chlamydial protease-like activity factor responsible for the degradation of host transcription factors. J Exp Med 193, 935-942.

Zhou, J. \& Xu, Z. (2005). The structural view of bacterial translocation-specific chaperone SecB: implications for function. Mol Microbiol 58, 349-357.

Edited by: K. Fields 